\title{
Spatial distribution of tuberculosis and its association with meteorological factors in mainland China
}

\author{
Yingjie Zhang ${ }^{1}$, Mengyang Liư ${ }^{2,3}$, Samuel S. Wu ${ }^{4}$, Hui Jiang ${ }^{5,6}$, Junjie Zhang ${ }^{7}$, Songwang Wang ${ }^{1}$, Wei Ma ${ }^{8}$, \\ Qihuan Li, ${ }^{2,3}$, Yuan Ma ${ }^{2,3}$, Yue Liu ${ }^{2,3}$, Wei Feng ${ }^{2,3}$, Endawoke Amsalü ${ }^{2,3}$, Xia Li ${ }^{9}$, Wei Wang ${ }^{10}$, Weimin Li $i^{3,5,6^{*}}$ and \\ Xiuhua Guo ${ }^{2,3^{*}}$
}

\begin{abstract}
Background: The incidence of tuberculosis (TB) remains high worldwide. Current strategies will not eradicate TB by 2035; instead, by 2182 is more likely. Therefore, it is urgent that new risk factors be identified.

Methods: An ecological study was conducted in 340 prefectures in China from 2005 to 2015. The spatial distribution of TB incidence was shown by clustering and hotspot analysis. The relationship between the distribution patterns and six meteorological factors was evaluated by the geographically weighted regression (GWR) model.

Results: During the 11 years of the study period, TB incidence was persistently low in the east and high in the west. Local coefficients from the GWR model showed a positive correlation between TB incidence and yearly average rainfall (AR) but a negative correlation with other meteorological factors. Average relative humidity (ARH) was negatively correlated with the incidence of TB in all prefectures $(p<0.05)$.
\end{abstract}

Conclusion: Meteorological factors may play an important role in the prevention and control of TB.

Keywords: Tuberculosis, Spatial distribution, Meteorological factors, Geographically weighted regression

\section{Background}

Tuberculosis (TB) is still one of the most infectious diseases worldwide with an estimated 10.0 million new cases and 1.3 million deaths according to the Global Tuberculosis Report from 2018 [1]. China is ranked second among the 30 high burden countries for TB and accounted for $9 \%$ of the world's cases. Previous studies indicated that TB epidemics were influenced by three principal aspects: environment, host (human) and pathogen $(M$. tuberculosis) $[2,3]$. The environmental aspect mainly refers to the social environment such as socioeconomic level, medical research and demographic factors [4-6]. The prevalence of TB in China has halved since 1991 due to the strategy of short-course chemotherapy following WHO guidelines to control TB [7].

\footnotetext{
* Correspondence: Iwm_18@aliyun.com; statguo@ccmu.edu.cn

${ }^{3}$ Beijing Municipal Key Laboratory of Clinical Epidemiology, Capital Medical University, Beijing 100069, China

${ }^{2}$ School of Public Health, Capital Medical University, Beijing 100069, China Full list of author information is available at the end of the article
}

However, if we rely only on the original strategy, the global TB epidemic, including China's epidemic, will not end by 2035, which is the aim of the World Health Organization (WHO)'s "End TB Strategy". To complete this aim as early as possible, and to further improve the Chinese TB control strategy, we should pay attention to new or previously neglected risk factors, such as weather [8]. At present, most studies have focused on the relationship between TB incidence and meteorological factors at the provincial level [9-11]. To further control TB incidence, we need to study the association between $\mathrm{TB}$ and meteorological factors from a narrower geographical area level. Additionally, biological research suggests that a temperature of $37^{\circ} \mathrm{C}$ and sufficient oxygen and water are favoured for the propagation of $M$. tuberculosis, but the pathogen is very sensitive to ultraviolet (UV) light, which is found in sunlight [12]. Therefore, it was speculated that meteorological factors may affect the tuberculosis incidence by affecting the growth and reproduction of $M$. tuberculosis to a certain extent. Therefore, the aim 
of this study was to explore whether meteorological factors from the prefecture level are potential determinants of TB incidence in China in order to provide a theoretical basis for the prevention and control measures of TB.

\section{Methods}

\section{Date sources}

All TB patients were from the Infectious Disease Reporting System (IDRS) and the Tuberculosis Information Management System (TBIMS) from the Chinese Center for Disease Control and Prevention (CCDC) [13, 14], which covers $99.6 \%$ of land area of China, including 340 prefectures (Fig. 1). We collected all TB case data from 1 January 2005 to 31 December 2015, including age, sex, address and some clinical diagnostic results.

We also obtained daily meteorological data for each prefecture during the study period from the China Meteorological Data Sharing Service System [15], including the average temperature $\left(\mathrm{AT},{ }^{\circ} \mathrm{C}\right)$, average relative humidity (ARH, \%), average wind speed (AWS, $\mathrm{m} / \mathrm{s}$ ), average atmospheric pressure (AAP, hPa), average rainfall $(\mathrm{AR}, \mathrm{mm})$ and average sunshine duration (ASD, h). In this study, average values from 2005 to 2015 were calculated from the daily values.

Additionally, the size of the population in each prefecture in every year was obtained from the Chinese
Statistical Bureau [16], which was used to calculate the TB incidence in each prefecture.

\section{Statistical analysis}

Frequently used descriptive epidemiologic methods were applied to analyse the distribution patterns of TB incidence. The Anselin Local Moran's I statistic and the Getis-Ord Gi* statistic were used to analyse the clustering of TB incidence and to identify TB incidence hotspots, respectively. The geographically weighted regression (GWR) model was used to explore the spatial variation of the relationship between TB incidence and meteorological factors. Spatial positions of the data were taken into consideration in the GWR model, so the local parameters changed with the spatial position [17]. Variance inflation factors (VIFs) were used to assess multicollinearity among the indicators [18], and we think that a collinearity problem does not exist among the meteorological factors.

A conventional GWR model can be described by the following equation:

$$
y_{j}=\beta_{0}\left(\mu_{j}, v_{j}\right)+\sum_{i=1}^{p} \beta_{i}\left(\mu_{j}, v_{j}\right) X_{i j}+\varepsilon_{j}
$$

where $\left(\mu_{\mathrm{j}}, v_{\mathrm{j}}\right)$ is the spatial coordinate of sample point $\mathrm{j}$; $\beta_{0}\left(\mu_{\mathrm{j}}, v_{\mathrm{j}}\right)$ and $\beta_{i}\left(\mu_{j}, v_{j}\right)$ are the regression constant and the

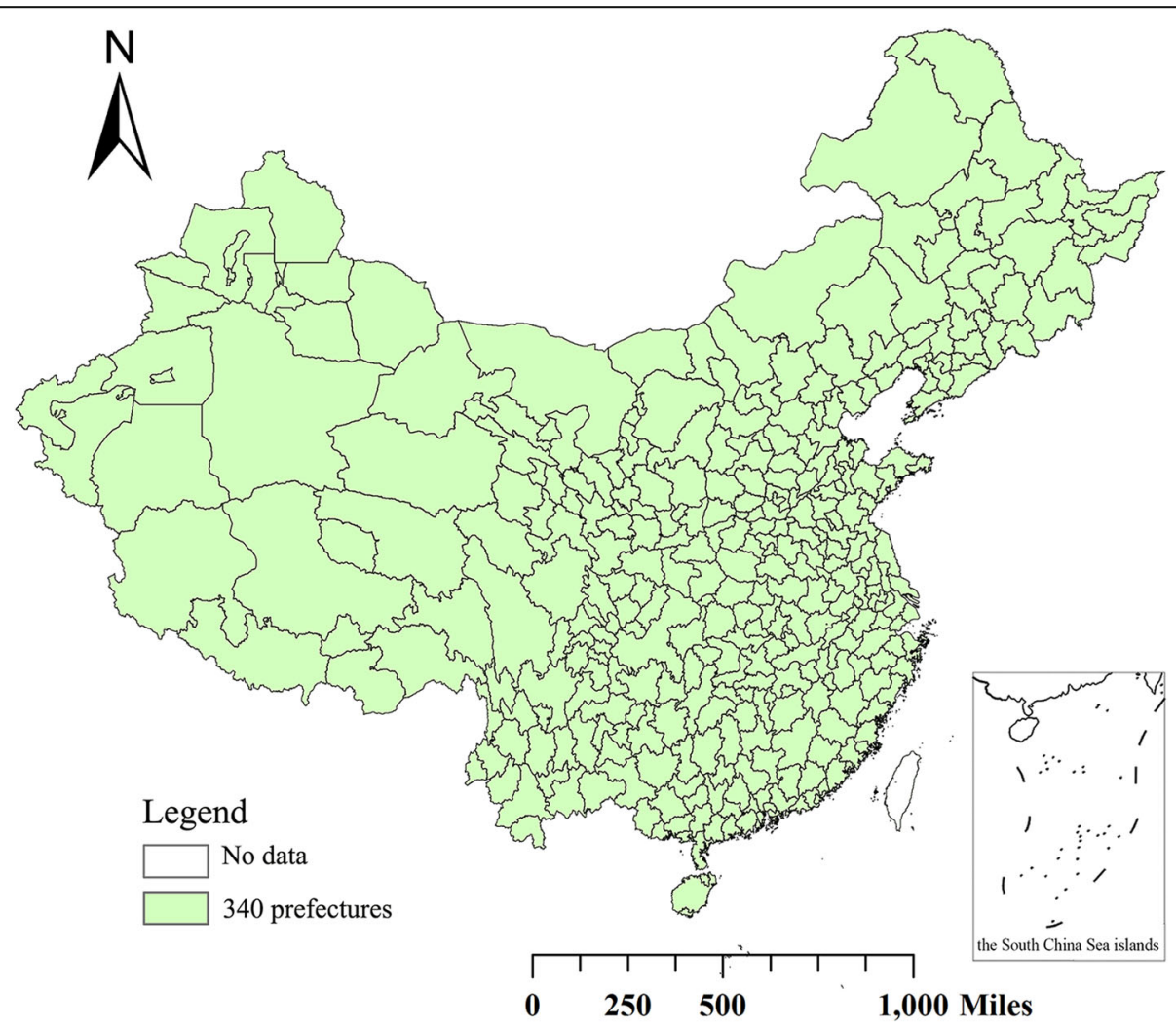

Fig. 1 The distribution of 340 prefectures in mainland China 
regression coefficient of sample point $\mathrm{j}$, respectively; and $\varepsilon_{\mathrm{j}}$ is the random error of the independent distribution. Considering that the samples were not regularly spaced in 340 prefectures, the adaptive bandwidth and Gaussian kernel were implemented to build the model, which provides the same number of samples for each local estimate and is based on the whitepaper for GWR $[19,20]$.

The GWR model was built with the GWR4.0 software. All statistical tests were two-sided, and $P$-values less than 0.05 were considered statistically significant.

\section{Results}

A total of 10,271,169 TB cases were reported from 1 January 2005 to 31 December 2015. After excluding cases with missing or abnormal values, our final sample included a total of $10,268,212$ TB cases.

\section{Spatial characteristics of TB incidence}

In general, the spatial analysis indicated an unbalanced geographical distribution of TB incidence in China, with high rate in the west and a low rate in the east between 2005 and 2015. The Xinjiang Uygur Autonomous Region had the highest incidence among 31 provinces. Specifically, Hotan Prefecture, Kashi Prefecture and Aksu Prefecture, located in the south of the Xinjiang Uygur Autonomous Region, had the highest TB incidence of over 180/100000. Additionally, some other prefectures adjacent to the border between the Tibet Autonomous Region and Gansu Province, Heilongjiang Province, Guizhou Province and Hainan Province also had a high TB incidence (Fig. 2b). In contrast, some areas in eastern China, such as Beijing, Tianjin, Shandong Province and Hebei Province, had relatively low TB incidence (Fig. 2a).
The clustering and hotspot analyses showed the prefectures with relatively high TB incidence, which coincided with the results in the thematic map and helped obtain a more accurate positioning. Six high-high clusters were found by the clustering analysis (Fig. 3a). Area (1) was in the south of Xinjiang and included three prefectures. Areas (2) and (3) included three prefectures in the north of Gansu and four prefectures in the east of Heilongjiang, respectively. Areas (4) included six prefectures adjacent to the border between Tibet and Qinghai Province, and Area (5) included seven prefectures within Hunan, Guizhou and Guangxi. Area (6) was in the south of Guangdong Province and consisted of seven prefectures (Fig. 3b).

\section{Association between TB incidence and meteorological factors}

The GWR model improved the fitness of the regression analysis (adj. $\left.\mathrm{R}^{2}=0.3523, \quad \mathrm{AICc}=3146.56\right) \quad$ compared with the ordinary least squares regression (OLS) model $\left(\right.$ adj. $R^{2}=0.1623$, AICc $\left.=3227.48\right)$. There were large differences in the distribution characteristics of meteorological factors in 340 prefectures (Additional file 1: Figure S1). The GWR model found that the influence effect and influence degree of six meteorological factors on TB incidence had significant spatial variability (Table 1 and Fig. 4). AR (Fig. 4e1) had a positive correlation with TB incidence from 2005 to 2015 while ARH (Fig. 4b1) and ASD (Fig. 4f1) were negatively correlated with TB incidence. The estimated local coefficients of AR, ARH and ASD ranged from 4.27 to $26.45,-19.76$ to -10.08 and -31.71 to -2.55 , respectively. Furthermore, ARH was statistically significant in all 340 prefectures $(P<0.05)$, while AR was significant for all

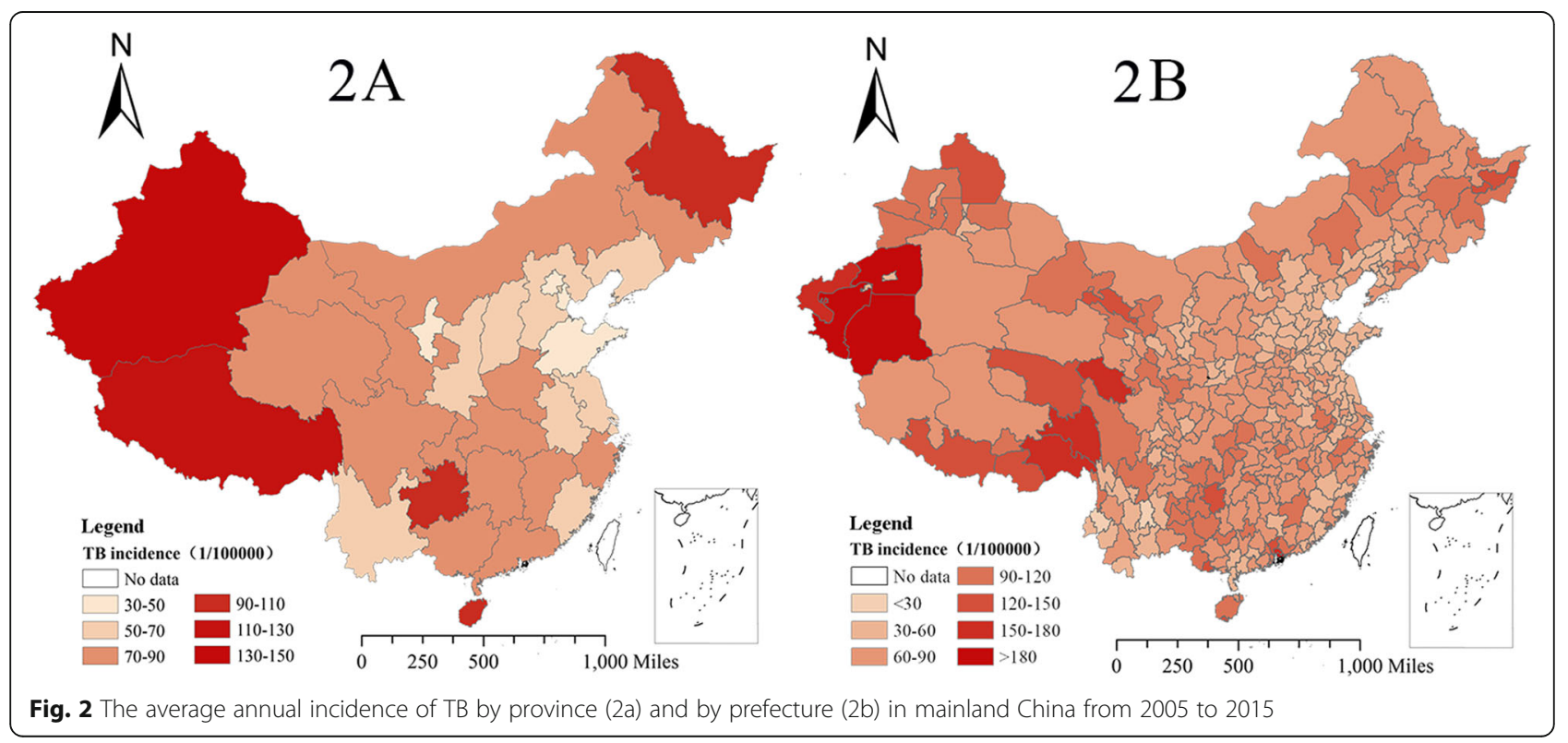




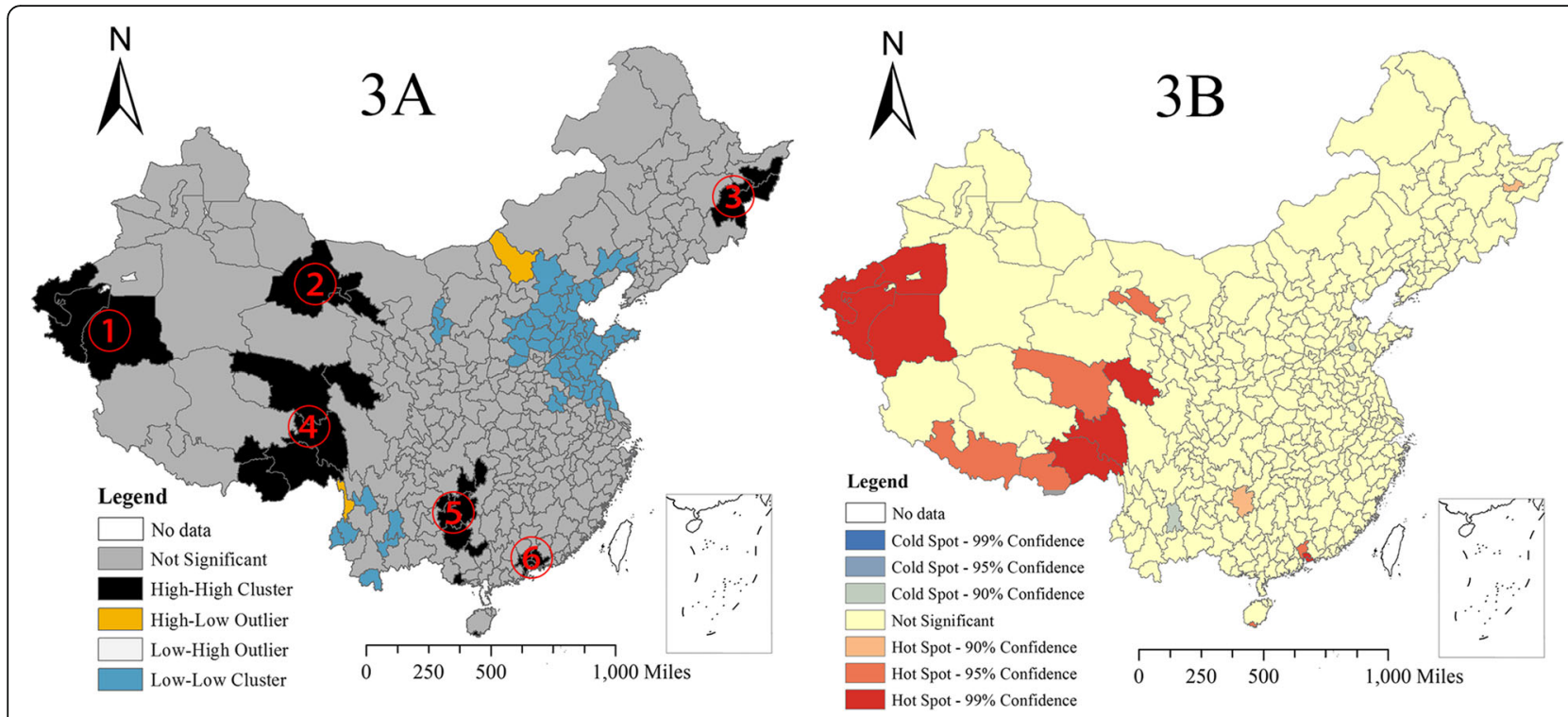

Fig. 3 Clusters from the Anselin Local Moran's I analysis (3a) and hotspots with the Getis-Ord Gi* statistic (3b) for TB incidence from 2005 to 2015

prefectures except some in the southeastern China, and ASD was significant for all prefectures except some in the western China. In addition, the relationships between the other three meteorological factors and TB incidence were not consistent across the study area. Specifically, AT was statistically significant only in north-eastern and central China (Fig. 4a1), and AWS and AAP were statistically significant in western China (Fig. 4c1 and Fig. 4d1, respectively), and all of them were negatively correlated.

\section{Discussion}

During 2005-2015 in mainland China, the spatial distribution presented a high incidence in the west and a low incidence in the east. The western region with high incidence included the Xinjiang Uygur Autonomous Region,

Table 1 The local GWR coefficient between reported TB incidence and meteorological factors in 340 prefectures in 2005-2015

\begin{tabular}{lllllll}
\hline Variable & Min & $P_{25}$ & $P_{50}$ & $P_{75}$ & Max & Range \\
\hline Intercept & 53.05 & 64.06 & 67.98 & 72.05 & 76.80 & 23.76 \\
AT $^{\mathrm{a}}$ & -28.71 & -17.93 & -9.82 & 1.21 & 12.84 & 41.55 \\
ARH $^{\mathrm{a}}$ & -19.76 & -18.52 & -17.93 & -16.55 & -10.08 & 9.68 \\
AWS $^{\mathrm{a}}$ & -8.58 & -1.27 & -0.51 & 0.87 & 2.99 & 11.57 \\
AAP $^{\mathrm{a}}$ & -7.78 & -5.05 & -3.91 & -0.75 & 10.49 & 18.27 \\
AR $^{\mathrm{a}}$ & 4.27 & 10.53 & 16.77 & 22.03 & 26.45 & 22.18 \\
ASD $^{\mathrm{a}}$ & -31.71 & -24.44 & -19.12 & -14.35 & -2.55 & 29.16 \\
\hline
\end{tabular}

${ }^{a}$ Table footnotes: ${ }^{a} A T$ (centigrade degree) Average temperature from 2005 to $2015,{ }^{a} A R H$ (\%) Average relative humidity from 2005 to $2015,{ }^{a}$ AWS (m/s) Average wind speed from 2005 to $2015 ;{ }^{\mathrm{a}} A A P(\mathrm{hPa})$ Average air pressure from 2005 to $2015,{ }^{\mathrm{a}} A R(\mathrm{~mm})$ Average rainfall from 2005 to 2015, ${ }^{\mathrm{a}} A S D$ (h) Average sunshine duration from 2005 to 2015 the Tibet Autonomous Region, Heilongjiang Province and Gansu Province. In contrast, the eastern region including Beijing, Tianjin and Shandong Province enjoyed a low TB incidence. The possible reasons for the geographic differences are as follows: first, the western region has a relatively underdeveloped economy, poor medical resources, a low educational level and a high proportion of minorities [21]; in contrast, eastern China has a relatively developed economy, adequate medical resources, a high educational level and a good living and work environment contribute to the low TB incidence. A recent survey of tuberculosis hospitals in China found that $34.6 \%$ of TB patients lived in western China, but only $12.8 \%$ of hospitals and $14.8 \%$ of beds were located in that region in 2010 [4]. In addition to the environment (socioeconomic) and host factors, we can also analyse these factors to observe how they relate to the pathogenic bacteria. $M$. $t u$ berculosis lineage 2 was dominant in the eastern region, while $M$. tuberculosis lineages 2, 3 and 4 were present in the western region [22, 23]. Many studies have suggested that $M$. tuberculosis lineage 2 is more pathogenic than other lineages [22] but the western China presented a more serious condition than the east, which implies that the environment may play a more important role in TB onset than the pathogen itself, so it is meaningful to conduct research on the meteorological factors. Additionally, from the cluster and hotspot analyses, we found that six areas (Fig. 3a) (four areas were in the west (1), (2), (4) and (5)), one was east of Heilongjiang (3), one was in the Zhujiang Delta, Guangdong (6)) had high TB incidence, which is the key areas for TB control and provention.

A correlation analysis with the GWR model showed that AR had a positive correlation with $\mathrm{TB}$ incidence. 


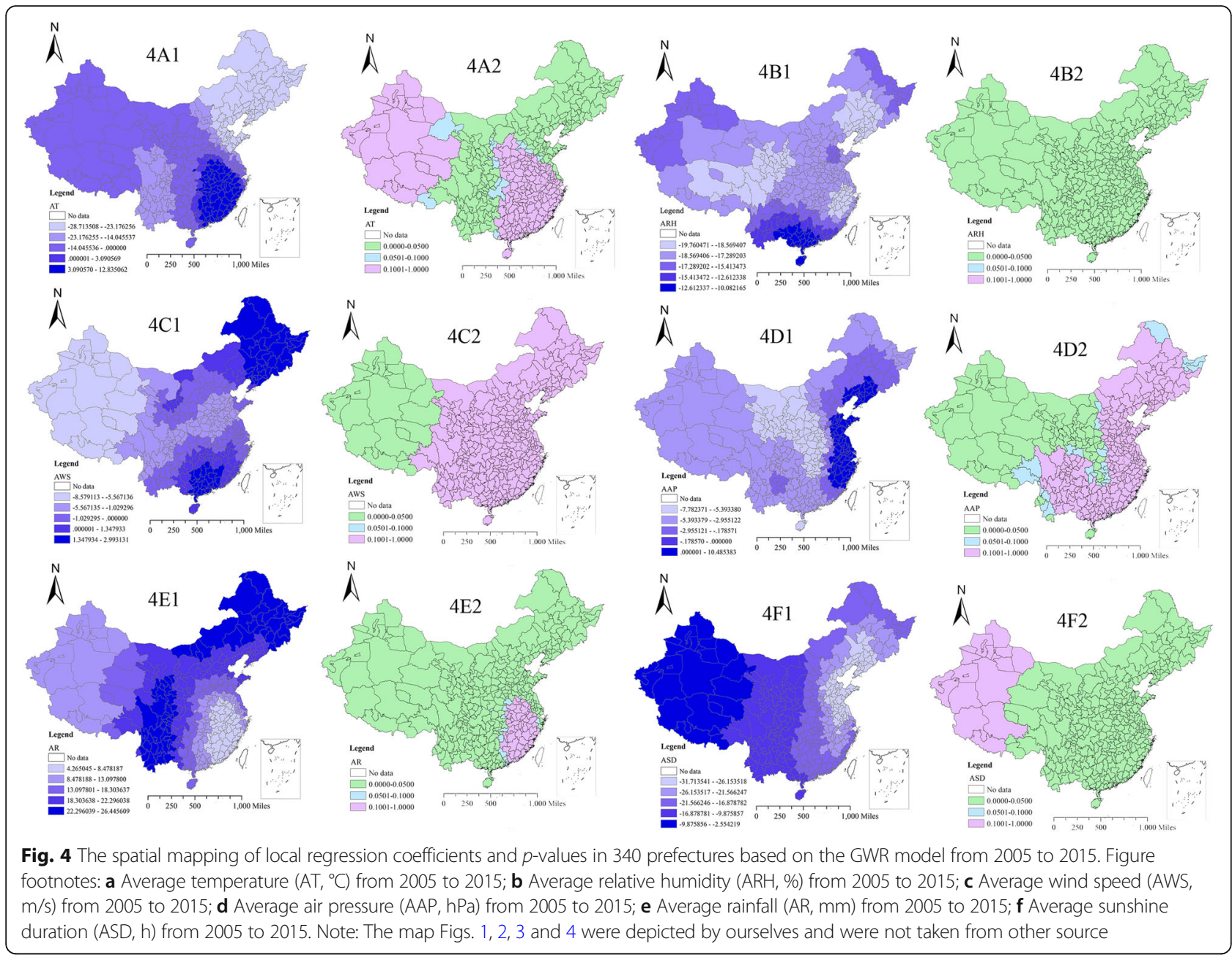

The increased rainfall may create a suitable environment for the growth and reproduction of M. tuberculosis [10, 24]. However, a negative correlation was observed between ASD and TB incidence. For ASD, on the one hand, a long sunshine duration with large amounts of UV light would restrict the development of $M$. tuberculosis [25]. On the other hand, the UV light could help the synthesis of vitamin D, which could protect people from TB to a certain extent [26, 27]. Additionally, biological research [12] support our study of meteorological factors across the country; that is, TB incidence is positively correlated with the average precipitation and is negatively corrected with the average sunshine duration.

M. tuberculosis is the pathogen of human $\mathrm{TB}$, and humans are hosts. The time from inhalation of M. tuberculosis to onset is long for humans, and meteorological factors are not relevant at this stage. However, meteorological factors may play an important role when TB patients expel $M$. tuberculosis into the surrounding environment by spitting. Our study also suggested that ARH is negatively correlated with the incidence of TB in the whole country $(p<0.05)$. In aerosol form only, $M$. tuberculosis can be inhaled into the lung to cause TB. Humanity has reduced dust emissions and the formulation of aerosols, and has thus reduced the spread of TB. In addition, TB incidence was associated with AWS and AAP in many prefectures. The higher AWS could accelerate ventilation, dilute the concentration of bacteria and help reduce the risk of becoming infected. For the AAP, increased atmosphere flow usually forms from high air pressure regions to low air pressure regions, so the mechanism of negative correlation between air pressure and TB incidence may be similar to wind speed, but further explorations are needed.

There are some limitations of the study. First, the potential for under-reporting of cases is inevitable in surveillance data, which is dependent on healthcare-seeking behaviours. In addition, if there is less healthcare available in certain areas, one might expect less reporting, so TB incidence would be underestimated. Second, this is a cross-sectional study with all data collapsed from 2005 to 2015 , so the time effects, such as a lag effect, were 
ignored. Third, we took only meteorological factors into consideration, and some other risk factors associated with TB incidence, such as healthcare access, socioeconomic status and individual-level factors that correlate with geography were not considered due to the unavailability of data. Therefore, further studies should control for other factors affecting TB at a more detailed time scale. Moreover, an ecological study cannot provide conclusive results but can only generate and develop hypotheses.

\section{Conclusion}

In this study, we found that meteorological factors may play an important role in TB incidence in many prefectures in mainland China. Therefore, the prevention and control strategies of TB should take meteorological factors into account. Next, we will proceed to further study about meteorological factors according to the results of this study.

\section{Additional file}

Additional file 1: Table S1. The number of reported TB cases in 31 provinces of mainland China, 2005-2015. Figure S1. Spatial distribution of six meteorological factors in 340 prefectures from 2005 to 2015. (DOC $1020 \mathrm{~kb}$ )

\section{Abbreviations}

AAP: Average atmospheric pressure; AR: Average rainfall; $\mathrm{ARH}$ : Average relative humidity; ASD: Average sunshine duration; AT: Average temperature; AWS: Average wind speed; CCDC: Chinese Center for Disease Control and Prevention; GWR: Geographically weighted regression; IDRS: Infectious Disease Reporting System; OLS: Ordinary least squares regression; TB: Tuberculosis; TBIMS: Tuberculosis Information Management System; UV: Ultraviolet; VIF: Variance inflation factor; WHO: World Health Organization

\section{Acknowledgements}

The authors thank Liping Zhu from Renmin University of China for the model optimization.

\section{Funding}

This work was supported by the National Science and Technology Major Project (2018ZX10302302-001-004). The funders had no role in study design, data collection and analysis, decision to publish, or preparation of the manuscript.

\section{Availability of data and materials}

The datasets generated and analysed during the current study are available in the Infectious Disease Reporting System (IDRS) and the Tuberculosis Information Management System (TBIMS) of the Chinese Center for Disease Control and Prevention (CCDC).

\section{Authors' contributions}

$Y Z$ had full access to all of the data in the study and wrote the manuscript. $W L$ and $X G$ take responsibility for the integrity of the data and the accuracy of the data analysis. ML, SW, HJ, WM, YM, QL, EA, WF and YL contributed substantially to the study design, data analysis and interpretation. SW, JZ, WW and $X L$ reviewed and revised the manuscript critically for important intellectual content. All authors read and approved the final manuscript.

\section{Ethics approval and consent to participate}

Tuberculosis is one of the Legal Infectious Diseases. It is mandated that each case of TB must be reported online within $24 \mathrm{~h}$ after diagnosis according to the Law of the People's Republic of China on the Prevention and Treatment of Infectious Diseases. Local CDC collected the local TB case and CCDC pooled all the cases together. So the ethical approval is unnecessary in this study.
It requires some administrative permissions to access the raw TB data. So we have applied the data and gained access to it from Chinese Center for Disease Control and Prevention.

\section{Consent for publication}

Not applicable.

\section{Competing interests}

The authors declare that they have no competing interests.

\section{Publisher's Note}

Springer Nature remains neutral with regard to jurisdictional claims in published maps and institutional affiliations.

\section{Author details}

${ }^{1}$ Chinese Center for Disease Control and Prevention, Beijing 102206, China. ${ }^{2}$ School of Public Health, Capital Medical University, Beijing 100069, China. ${ }^{3}$ Beijing Municipal Key Laboratory of Clinical Epidemiology, Capital Medical University, Beijing 100069, China. ${ }^{4}$ Department of Statistics, University of Florida, Gainesville, FL 32610-7450, USA. ${ }^{5}$ National Tuberculosis Clinical Lab of China, Beijing Chest Hospital, Capital Medical University, Beijing 101149, China. ${ }^{6}$ Beijing Key Laboratory of Drug Resistance Tuberculosis, Beijing Tuberculosis and Thoracic Tumor Research Institute, Beijing 101149, China. ${ }^{7}$ School of life sciences, Beijing Normal University, Beijing 100875, China. ${ }^{8}$ Institute of statistics and big data, Renmin University of China, Beijing 100872, China. ${ }^{9}$ Department of Mathematics and Statistics, La Trobe University, Bundoora, Victoria 3086, Australia. ${ }^{10}$ School of Medical Sciences and Health, Edith Cowan University, WA6027, Perth, Australia.

Received: 21 October 2018 Accepted: 22 April 2019

Published online: 03 May 2019

\section{References}

1. World Health Organization. Global tuberculosis report 2018: WHO press; 2018. Available at: http://www.who.int/tb/publications/global_report/en/. Accessed 26 Sep 2018

2. Comas I, Gagneux S. A role for systems epidemiology in tuberculosis research. Trends Microbiol. 2011;19(10):492-500.

3. Coscolla M, Gagneux S. Does M. tuberculosis genomic diversity explain disease diversity? Drug Discov Today Dis Mech. 2010;7(1):e43-59.

4. Du J, Pang Y, Liu Y, Mi F, Xu S, Li L. Survey of tuberculosis hospitals in China: current status and challenges. PLoS One. 2014;9(11):e111945.

5. Wang W, Jin YY, Ci Y, Ahan A, Cao MQ. Local spatial variations analysis of smear-positive tuberculosis in Xinjiang using geographically weighted regression model. BMC Public Health. 2016;16(1):1058.

6. Wubuli A, Xue F, Jiang D, Yao X, Upur H, Wushouer Q. Socio-demographic predictors and distribution of pulmonary tuberculosis in Xinjiang, China: a spatial analysis. PLoS One. 2015;10(12):e0144010.

7. Chen XY, Duanmu H, Wan LY, Zhao FZ, Wang LX, Tu DH, Du X, Kim SJ, Watt C, Williams B. The effect of tuberculosis control in China. Lancet. 2004; 364(9432):417-22.

8. Raviglione MC, Ditiu L. Setting new targets in the fight against tuberculosis. Nat Med. 2013;19(3):263.

9. Guo C, Du Y, Shen SQ, Lao XQ, Qian J, Ou CQ. Spatiotemporal analysis of tuberculosis incidence and its associated factors in mainland China. Epidemiol Infect. 2017;145(12):2510-9.

10. Cao K, Yang K, Wang C, Guo J, Tao L, Liu Q, Gehendra M, Zhang Y, Guo X. Spatial-temporal epidemiology of tuberculosis in mainland China: an analysis based on bayesian theory. Int J Environ Res Public Health. 2016;13:5.

11. Li XX, Wang LX, Zhang J, Liu YX, Zhang H, Jiang SW, Chen JX, Zhou XN. Exploration of ecological factors related to the spatial heterogeneity of tuberculosis prevalence in P. R. China. Glob Health Action. 2014;7:23620.

12. Yan BY, Duanmu HJ. Tuberculosis. 1st ed. Beijing: Beijing Publishing House; 2003.

13. Huang F, Cheng S, Du X, Chen W, Scano F, Falzon D, Wang L. Electronic recording and reporting system for tuberculosis in China: experience and opportunities. J Am Med Inform Assoc. 2014;21(5):938-41.

14. Wang L, Liu X, Huang F, Hennig C, Uplekar M, Jiang S. Engaging hospitals to meet tuberculosis control targets in China: using the internet as a tool to put policy into practice. Bull World Health Organ. 2010;88(12):937-42. 
15. China Meteorological Data Sharing Service System. National Meteorological Information Center, Beijing. 2005. http://data.cma.cn. Accessed 1 Jan 2005.

16. Prefecture-level municipal population database. National Bureau of Statistics of China, Beijing. 1952. http://data.stats.gov.cn. Accessed 1 Jan 2005.

17. Fotheringham AS, Brunsdon C, Charlton M. Geographically weighted regression: the analysis of spatially varying relationships. 1rd ed. Chichester: Wiley; 2002.

18. Belsley DA, Kuh E, Welsch RE. Regression diagnostics: identifying influential data and sources of collinearity, 1rd ed. New York: Wiley; 1980.

19. Charlton M. Geographically Weighted Regression, White Paper. 1rd ed. Maynooth: National Centre for Geocomputation, National University of Ireland Maynooth; 2009.

20. Nakaya T. GWR4 User Manual: Department of Geography, Ritsumeikan University; 2014. http://www.st-andrews.ac.uk/geoinformatics/wpcontent/ uploads/GWR4manual. Accessed 12 Mar 2014

21. Wang L, Zhang H, Ruan Y, Chin DP, Xia Y, Cheng S, Chen M, Zhao Y, Jiang S, Du X, et al. Tuberculosis prevalence in China, 1990-2010; a longitudinal analysis of national survey data. Lancet. 2014;383(9934):2057-64.

22. Chen H, He L, Cai C, Liu J, Jia J, Ma L, Huang H, Wang L, Ni X, Gao J, et al. Characteristics of distribution of mycobacterium tuberculosis lineages in China. Sci China Life Sci. 2018;61(6):651-9.

23. Chen H, He L, Huang H, Shi C, Ni X, Dai G, Ma L, Li W. Mycobacterium tuberculosis lineage distribution in Xinjiang and Gansu provinces. China Sci Rep. 2017;7(1):1068.

24. Desalu $\mathrm{OO}$. Seasonal variation in hospitalisation for respiratory diseases in the tropical rain forest of south western Nigeria. Niger Postgrad Med J. 2011;18(1):39-43.

25. Peccia J, Hernandez M. UV-induced inactivation rates for airborne mycobacterium bovis BCG. J Occup Environ Hyg. 2004;1 (7):430-5.

26. Hong JY, Kim SY, Chung KS, Kim EY, Jung JY, Park MS, Kim YS, Kim SK, Chang J, Kang YA. Association between vitamin D deficiency and tuberculosis in a Korean population. Int J Tuberc Lung Dis. 2014;18(1):73-8.

27. Selvaraj P, Harishankar M, Afsal K. Vitamin D: immuno-modulation and tuberculosis treatment. Can J Physiol Pharmacol. 2015;93(5):377-84.

Ready to submit your research? Choose BMC and benefit from:

- fast, convenient online submission

- thorough peer review by experienced researchers in your field

- rapid publication on acceptance

- support for research data, including large and complex data types

- gold Open Access which fosters wider collaboration and increased citations

- maximum visibility for your research: over $100 \mathrm{M}$ website views per year

At $\mathrm{BMC}$, research is always in progress.

Learn more biomedcentral.com/submissions 